\title{
Belgeo
}

Revue belge de géographie

1-2 | 2012

Inaugural issue

\section{Vivre la frontière : les pratiques spatiales transfrontalières dans l'Arc jurassien franco-suisse}

Living the border : cross-border spatial practices in the French-Swiss Jura region

\section{Yann Dubois et Patrick Rérat}

\section{OpenEdition}

\section{Journals}

Édition électronique

URL : http://journals.openedition.org/belgeo/6249

DOI : $10.4000 /$ belgeo.6249

ISSN : 2294-9135

Éditeur :

National Committee of Geography of Belgium, Société Royale Belge de Géographie

Référence électronique

Yann Dubois et Patrick Rérat, « Vivre la frontière : les pratiques spatiales transfrontalières dans l'Arc jurassien franco-suisse », Belgeo [En ligne], 1-2 | 2012, mis en ligne le 15 décembre 2012, consulté le 01 mai 2019. URL : http://journals.openedition.org/belgeo/6249; DOI : 10.4000/belgeo.6249

Ce document a été généré automatiquement le 1 mai 2019.

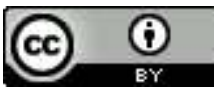

Belgeo est mis à disposition selon les termes de la licence Creative Commons Attribution 4.0 International. 


\section{Vivre la frontière : les pratiques spatiales transfrontalières dans l'Arc jurassien franco-suisse}

Living the border : cross-border spatial practices in the French-Swiss Jura region Yann Dubois et Patrick Rérat

\section{Introduction}

1 Les frontières nationales ont constamment été redéfinies au cours de l'histoire. Davantage que leur tracé, ce sont leurs rôles et fonctions qui ont évolué selon certains contextes historiques, politiques, économiques et sociaux (Anderson et O'Dowd, 1999; Newman, 2006; Wastl-Walter, 2009 et 2011; Nelles et Walther, 2011; Rérat et WastlWalter, 2011). Lors des dernières décennies du XX siècle, d'importants changements géopolitiques et économiques ont contribué à une redéfinition des frontières nationales. La chute du Mur de Berlin a marqué l'effondrement du bloc soviétique et la fin d'un monde bipolaire. Des entités supranationales ont été créées au premier rang desquelles se trouve l'Union européenne. Parallèlement, cette période s'est caractérisée par la mondialisation, phénomène protéiforme défini comme une compression de l'espacetemps (grâce entre autres aux technologies de l'information), la conscience d'une condition globale et une interdépendance accrue entre les économies notamment en termes de flux de capitaux, de personnes, d'informations et de marchandises (Held et MacGrew, 2003).

2 Ces évolutions ont été interprétées par les tenants d'une approche qualifiée de "strong globalization" (Diener et Hagen, 2009), comme annonçant la disparition des frontières nationales, l'avènement d'un monde sans frontière ("borderless world") et la fin de la géographie (Ohmae, 1990 ; O'Brien, 1992). En réaction à ces discours, d'autres chercheurs ont adopté une approche plus mesurée ("weak globalization") en arguant que certaines frontières deviendront de plus en plus perméables et constitueront des sites d'interaction mais que d'autres resteront des barrières (Diener et Hagen, 2009). Deux arguments sont 
principalement avancés pour justifier ce dernier positionnement. D'une part, de nombreuses frontières nationales ont été nouvellement créées ou ont été renforcées dans leur rôle de contrôle notamment dans la période post-11 septembre. À titre d'illustration, la construction européenne (et l'affaiblissement des frontières internes) va de pair avec la constitution d'une "forteresse européenne" (soit le renforcement des frontières externes). D'autre part, les activités humaines continuent de se dérouler à l'intérieur de territoires relativement bien définis (Newman, 2006). Ainsi, la période actuelle se caractériserait moins par un processus de déterritorialisation mais davantage par un processus de reterritorialisation du monde (Wastl-Walter, 2011).

Situé dans le prolongement de cette deuxième perspective, cet article se focalise sur l'impact de la redéfinition d'une frontière nationale sur les pratiques des habitants de la région qu'elle traverse, et sur la manière dont ces dernières contribuent à redéfinir la signification de la frontière (voir également Rérat et Wastl-Walter, 2011). Cette étude entend plus particulièrement répondre à deux objectifs: (1) mesurer l'existence et l'intensité de certaines pratiques spatiales transfrontalières et (2) déterminer les logiques sous-jacentes à ces pratiques en lien avec la frontière. Elle prend comme étude de cas l'Arc jurassien franco-suisse, soit un exemple de frontière de plus en plus perméable à différentes formes de mobilité. En effet, bien que la Suisse ne fasse pas partie de l'Union européenne, de nombreux accords assurent une circulation aisée des biens, services, personnes et capitaux entre ces deux entités.

\section{Eléments théoriques}

\section{Conceptualiser la frontière}

4 Quatre fonctions majeures des frontières nationale sont traditionnellement distinguées (Blake, 2000): elles délimitent la souveraineté entre États; elles permettent aux gouvernements de contrôler les mouvements et flux qui les traversent ; elles constituent une enveloppe protectrice et militaire afin de défendre le territoire national ; finalement, ces trois fonctions deviennent des instruments pour construire l'identité nationale.

Les frontières ont ainsi souvent été présentées comme étant tour à tour barrière ou interface, coupure ou couture. Toutefois, la plupart des frontières présentent simultanément les deux visages. Il a ainsi été proposé de les conceptualiser comme un ensemble de règles, de normes et de procédures qui régulent et contrôlent leurs effets sur les acteurs sociaux, politiques et économiques (Nelles et Walther, 2011). La frontière apparait ainsi comme une construction politique évolutive qui a un triple impact sur le développement territorial des régions frontalières et sur les pratiques spatiales de leurs habitants.

6 Premièrement, la frontière sépare deux systèmes institutionnels divergeant sur des aspects tels que la législation, la fiscalité, le fonctionnement de l'administration, le système scolaire, etc. Dans ce sens, la frontière "met de la distance dans la proximité" (Groupe frontière, 2004), que ce soit dans des éléments aussi divers que la collaboration entre communes proches, l'organisation de réseaux de transports publics ou encore en termes d'identité régionale. Deuxièmement, la frontière permet aux États de réguler les flux entrants et sortants et de déterminer les formes de mobilité (de personnes, de marchandises, etc.) autorisées à la traverser. Troisièmement, la frontière crée des différentiels de prix, de salaires, de normes légales, etc. L'exploitation de ces différentiels 
est à l'origine de stratégies ou de tactiques et génère des flux, licites ou non, répondant souvent à une logique d'opportunité (Groupe frontière, 2004).

7 Une autre manière d'aborder les impacts de la frontière et l'interaction entre territoires frontaliers est d'utiliser la notion d'intégration (De Oliveira, 2009 ; Reitel, 2007 ; Sohn et Walther, 2009). Ces auteurs distinguent plus particulièrement l'intégration formelle et l'intégration fonctionnelle.

8 L'intégration formelle, de nature politique, concerne "la forme et l'intensité des interactions entre les acteurs politiques éventuellement disposés à collaborer" (Sohn et Walther, 2009, p. 53). Elle regroupe les accords signés entre États pour régir une frontière et sa perméabilité mais aussi, à l'échelle régionale, les différentes formes de collaborations transfrontalières. Les deux échelles sont parfois étroitement liées. C'est le cas du programme Interreg institué dans les années 1990 par la Commission européenne et qui soutient financièrement des projets visant à encourager la collaboration transfrontalière au sein de l'Union européenne ainsi qu'avec des pays adjacents comme la Suisse.

9 L'intégration fonctionnelle renvoie quant à elle à "la forme et à l'intensité des interactions socio-économiques observées de part et d'autre d'une frontière" (Sohn et Walther, 2009, p.53). Elle rassemble les pratiques spatiales transfrontalières et les diverses formes de mobilité traversant la frontière. Ces échanges sont soumis à des cadres légaux mais il peut également exister une certaine permissivité voire illégalité (contrebande par exemple).

\section{Pratiques spatiales et logiques d'action}

10 Cet article entend analyser les pratiques spatiales transfrontalières (qui traduisent le degré d'intégration fonctionnelle) dans un contexte marqué par une intégration formelle croissante (ainsi que nous le détaillerons au point suivant). Les pratiques spatiales qui nous intéressent ici sont des actions plus ou moins routinières et régulières qu'exercent les habitants dans leur temps libre, liées à leurs conduites de consommation ou de sociabilité (Juan, 1997). Cet espace "routinier", ces "sentiers de la vie quotidienne" pour reprendre l'expression de Giddens (1984), n'englobent que rarement un seul lieu mais plutôt une multitude de lieux qui correspondent à différentes pratiques (travail, loisirs, habitat, etc.).

11 Le premier objectif de l'article consiste à aborder la dimension transfrontalière de l'espace de vie d'habitants proches de la frontière. Cela implique de s'intéresser à la mobilité régulière entre les endroits où se déroulent les pratiques spatiales suivantes : les réseaux sociaux, les loisirs et les achats. Le second objectif est de comprendre les logiques sous-jacentes aux pratiques spatiales transfrontalières (motivations et freins). Nous reprenons ici la typologie développée par Bolzman et Vial (2007) et qui distingue trois grandes logiques : économique et calculatrice, utilitaire et socio-culturelle.

12 La logique économique et calculatrice renvoie en premier lieu dans un contexte transfrontalier aux différentiels de coût de la vie et de pouvoir d'achat (niveau des prix et des salaires, évolution du taux de change, existence de taxes sur certains biens de consommation, etc.). Elle relève l'existence d'opportunités créées par la frontière et de pratiques relevant d'économies d'arbitrage (De Oliveira, 2009). La logique utilitaire comprend des aspects d'ordre pratique tels que la proximité et l'accessibilité (distance, 
transports publics, etc.) mais aussi l'offre de biens et services (choix, qualité). La logique socio-culturelle regroupe quant à elle des facteurs tels que l'habitude, les préférences, la différence de mentalité ou le degré de connaissance du pays voisin. Bolzman et Vial (2007) ont par exemple montré l'importance de la socialisation secondaire dans le cas des travailleurs frontaliers. Il s'agit de certaines adaptations progressives des attitudes et des comportements au milieu dans lequel un individu est inséré. Ainsi, le contact avec des collègues de travail suisses permettrait aux frontaliers de bénéficier d'informations plus précises et de se familiariser davantage avec les biens et services helvétiques.

Avant de présenter des résultats empiriques sur les pratiques spatiales transfrontalières, la partie suivante décrit la démarche de recherche et détaille plus particulièrement le périmètre d'étude et les méthodes employées.

\section{Démarche de recherche}

\section{Présentation du périmètre d'étude}

Cette étude prend place dans l'Arc jurassien, massif montagneux situé à cheval sur la frontière franco-suisse (figure 1). En termes d'intégration formelle, les dispositions en vigueur régulant la circulation des personnes et des biens pour la consommation personnelle entre la France et la Suisse permettent un passage aisé de la frontière, tant au niveau du travail, que pour le reste de la vie quotidienne (consommation, loisirs, etc.) ${ }^{1}$. En termes d'intégration fonctionnelle, des différentiels relativement importants en matière de salaires et de coût de la vie (plus élevés en Suisse) induisent de nombreux flux (Moine, 2003). Les principaux sont les travailleurs frontaliers (avec une concentration résidentielle autour des points de franchissement de la frontière sur sol français) (Chevailler, 1990 ; Crevoisier et al., 2006; Garufo, 2009), les relations de sous-traitance (pièces de microtechnique conçues en Suisse, usinées en France et définitivement assemblées en Suisse pour bénéficier du "Swiss made") (Munz, 2011), le tourisme d'achat (de la part des Suisses et orienté vers les commerces et services médicaux). Certains flux résidentiels de part et d'autre de la frontière ont également été analysés (Rérat $e t$ al., 2011). Les autres pratiques transfrontalières, dont celles qui nous intéressent ici, sont en revanche peu connues.

Cette étude s'intéresse plus particulièrement à deux communes françaises : Morteau et Villers-le-Lac (figure 2). Comptant respectivement 6824 et 4537 habitants, ces communes se trouvent à quelques kilomètres de la frontière suisse. L'Arc jurassien est caractérisé par l'absence de grands centres urbains, ceux-ci se trouvant plutôt dans les zones de plaine qui l'entourent (comme Besançon ou Lausanne). Autour des deux communes étudiées, les centres les plus importants sont Pontarlier (19450 habitants) du côté français, Le Locle (10 052) et La Chaux-de-Fonds ( 37 523) du côté helvétique².

Morteau et Villers-le-Lac entretiennent des relations privilégiées avec les villes suisses voisines du Locle et de La Chaux-de-Fonds, spécialisées dans l'horlogerie et la microtechnique, notamment en leur fournissant de nombreux travailleurs frontaliers. Selon le recensement de la population de l'INSEE de 2008, 44,9\% des actifs vivant à Morteau et 51,7 \% à Villers-le-Lac travaillent en Suisse. Ainsi, alors que Le Locle et La Chaux-de-Fonds constituent des centres d'emploi, les communes françaises proches peuvent être considérées comme des communes à vocation résidentielle. En outre, les 
différences de taille démographique se répercutent sur le niveau et la diversité des infrastructures et services.

Figure 1. L'Arc jurassien franco-suisse.

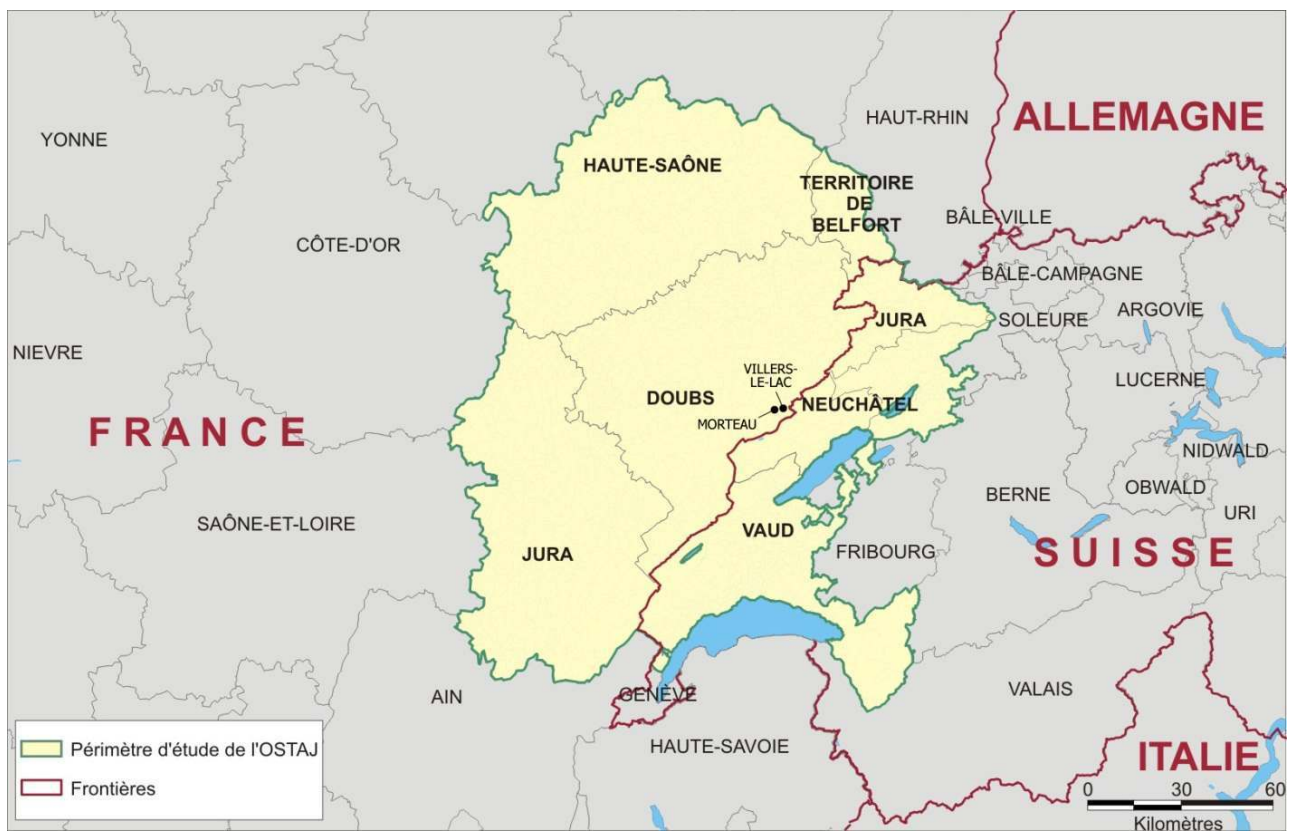

Source : Observatoire statistique transfrontalier de l'Arc jurassien - OSTAJ

Figure 2. Localisation des communes étudiées (reproduit avec l'autorisation de swisstopo ; BA12003).

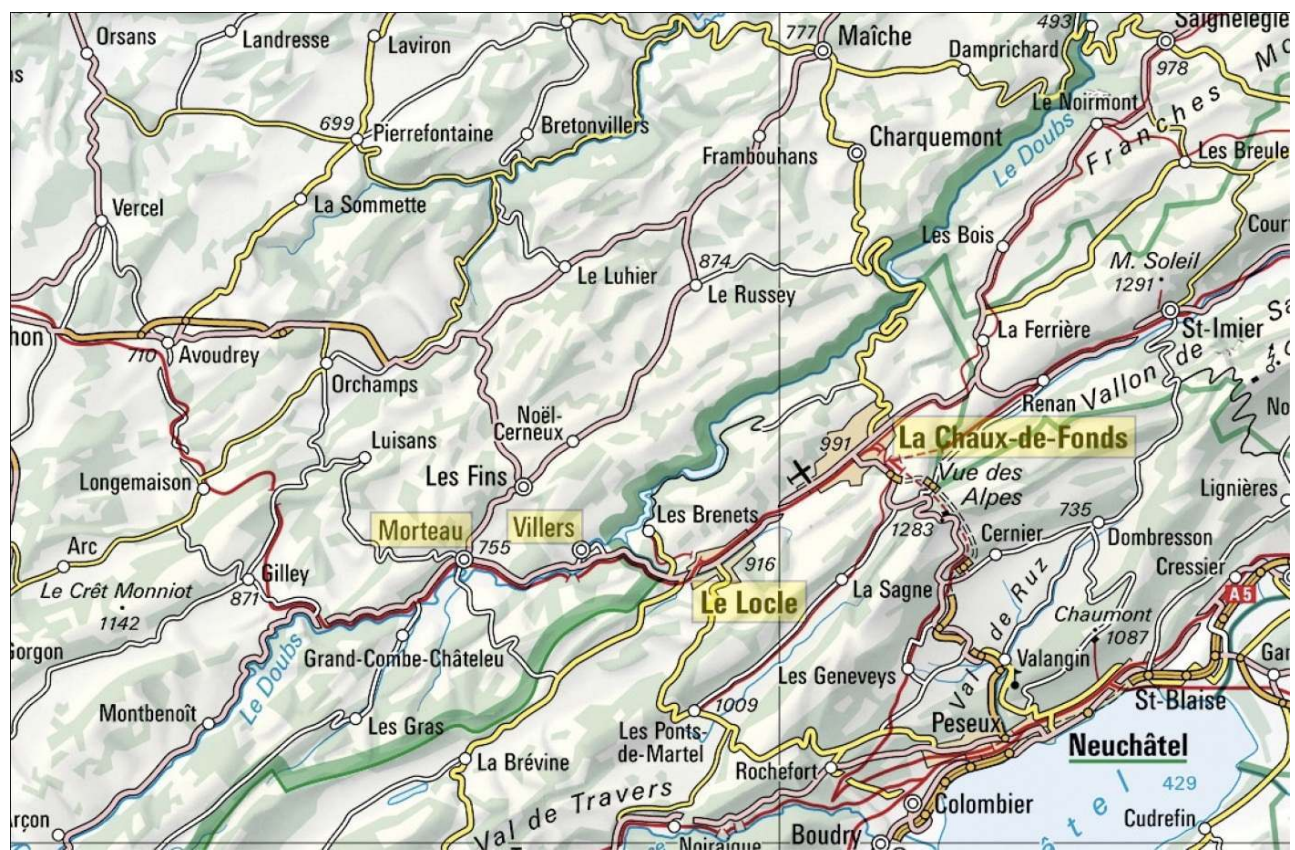




\section{Méthodologie}

17 Une enquête par questionnaire a été réalisée en 2011 auprès de la population de Morteau et Villers-le-Lac. Les personnes interrogées ont été identifiées par l'intermédiaire d'un annuaire réunissant les différents opérateurs téléphoniques (il n'a pas été possible d'obtenir les adresses auprès des communes). Certains biais peuvent découler d'une telle démarche: les personnes ne disposant pas d'un téléphone fixe n'y sont pas nécessairement enregistrées ; les jeunes vivant encore chez leurs parents n'y figurent pas, etc. Malgré cela, le nombre de numéros présents dans l'annuaire s'approche du nombre de ménages dans ces deux communes, ce qui semble confirmer son exploitabilité.

Morteau comptant 1,5 fois plus d'habitants que Villers-le-Lac, ce ratio a été reporté sur la taille de l'échantillon, soit 1200 questionnaires dans la première commune et 800 dans la seconde. Afin de disposer d'un échantillon représentatif, un tirage systématique a été effectué en sélectionnant une personne sur deux dans la base de données. Un taux de réponse de $36.2 \%$ a été obtenu ${ }^{3}$.

19 Le questionnaire portait sur le profil des personnes (formation, âge, lieu de travail, etc.) ainsi que sur les trois pratiques spatiales étudiées : les réseaux sociaux (cercle d'amis et vie associative), les loisirs et les achats. Pour ces deux derniers étaient abordés la localisation des pratiques et les facteurs les influençant. Plusieurs endroits étaient prévus dans le questionnaire pour accueillir des commentaires, ce qui devait permettre de mieux cerner certains mécanismes.

\section{Présentation des résultats}

L'analyse des pratiques spatiales transfrontalières des habitants de Morteau et Villers-leLac est découpée en trois parties. La première décrit les pratiques spatiales retenues. La seconde distingue les pratiques des frontaliers de celles du reste de la population. La troisième explique quant à elle les mécanismes sous-jacents à ces pratiques en mettant en exergue ce qui incite la population à traverser la frontière ou au contraire ce qui l'en retient.

\section{Pratiques spatiales transfrontalières}

21 S'intéresser aux réseaux sociaux permet de prendre en compte une facette importante des interactions transfrontalières. En effet, avoir des amis dans le pays voisin ou participer à la vie associative locale témoigne d'une certaine intégration transfrontalière au-delà des différentiels induits par la frontière. Le premier indicateur retenu concerne la localisation du cercle d'amis (tableau 1)4. Plus de six personnes sur dix ont un réseau social situé (quasi) uniquement sur le versant français, alors que près de quatre sur dix déclarent avoir des amis des deux côtés de la frontière. Le second aspect est la participation à la vie associative $e^{5}$. Les sondés devaient indiquer s'ils fréquentaient des associations en France, en Suisse ou dans les deux pays. Parmi ceux qui participent à la vie associative ( $63,7 \%$ de l'échantillon), environ $60 \%$ le font uniquement en France, le reste étant membre d'une association en Suisse ou dans les deux pays. 
Tableau 1. Pratiques spatiales transfrontalières.

\begin{tabular}{|c|c|c|c|}
\hline & $\begin{array}{l}\text { En Suisse et en France/ } \\
\text { uniquement en Suisse }\end{array}$ & $\begin{array}{l}\text { Uniquement en } \\
\text { France }\end{array}$ & Total \\
\hline \multicolumn{4}{|l|}{ Réseaux sociaux : } \\
\hline Cercle d'amis & $38.1 \%$ & $61.9 \%$ & $\begin{array}{l}100 \% \\
(659)\end{array}$ \\
\hline Participation à des associations & $39.3 \%$ & $60.7 \%$ & $\begin{array}{l}100 \% \\
(427)\end{array}$ \\
\hline \multicolumn{4}{|l|}{$\underline{\text { Loisirs }}$} \\
\hline Promenade & $72.6 \%$ & $27.4 \%$ & $\begin{array}{l}100 \% \\
(576)\end{array}$ \\
\hline Bar, restaurant, discothèque & $64.0 \%$ & $36.0 \%$ & $\begin{array}{l}100 \% \\
(408)\end{array}$ \\
\hline $\begin{array}{l}\text { Théâtre, spectacle, concert, } \\
\text { musée, événement culturel }\end{array}$ & $60.0 \%$ & $40.0 \%$ & $\begin{array}{l}100 \% \\
(375)\end{array}$ \\
\hline Cinéma & $49.2 \%$ & $50.8 \%$ & $\begin{array}{l}100 \% \\
(364)\end{array}$ \\
\hline Piscine & $64.3 \%$ & $35.7 \%$ & $\begin{array}{l}100 \% \\
(339)\end{array}$ \\
\hline Sport, activité de plein-air & $62.6 \%$ & $37.4 \%$ & $\begin{array}{l}100 \% \\
(286)\end{array}$ \\
\hline $\begin{array}{l}\text { Evénement sportif (en tant que } \\
\text { spectateur) }\end{array}$ & $48.1 \%$ & $51.9 \%$ & $\begin{array}{l}100 \% \\
(189)\end{array}$ \\
\hline $\begin{array}{l}\text { Sport en salle (squash, tennis, } \\
\text { fitness, etc.) }\end{array}$ & $48.8 \%$ & $51.2 \%$ & $\begin{array}{l}100 \% \\
(129)\end{array}$ \\
\hline Patinoire & $71.4 \%$ & $28.6 \%$ & $\begin{array}{l}100 \% \\
(91)\end{array}$ \\
\hline \multicolumn{4}{|l|}{ Achats } \\
\hline Produits alimentaires & $62.5 \%$ & $37.5 \%$ & $\begin{array}{l}100 \% \\
(670)\end{array}$ \\
\hline Essence & $66.6 \%$ & $33.4 \%$ & $\begin{array}{l}100 \% \\
(670)\end{array}$ \\
\hline Cigarettes & $24.3 \%$ & $75.7 \%$ & $\begin{array}{l}100 \% \\
(670)\end{array}$ \\
\hline
\end{tabular}




\begin{tabular}{|l|l|l|l|}
\hline Vêtements/articles de sport & $58.4 \%$ & $41.6 \%$ & $\begin{array}{l}100 \% \\
670)\end{array}$ \\
\hline $\begin{array}{l}\text { Produits liés à l'équipement de la } \\
\text { maison (meubles, décorations) }\end{array}$ & $34.5 \%$ & $65.5 \%$ & $70.0 \%$ \\
\hline Montres/Bijoux & $30.0 \%$ & $\begin{array}{l}100 \% \\
(670)\end{array}$ \\
\hline $\begin{array}{l}\text { Électroménager/HIFI } \\
\text { Cosmétiques, bien-être, salon de } \\
\text { coiffure }\end{array}$ & $23.7 \%$ & $71.6 \%$ & $\begin{array}{l}100 \% \\
(670)\end{array}$ \\
\hline
\end{tabular}

Note : la localisation du cercle d'amis et des achats renvoie à l'ensemble de la population interrogée ; les chiffres relatifs à la participation à des associations et aux loisirs ne concernent que les personnes pratiquant ces activités.

Source : enquête par questionnaire, 2011

Neuf types de loisirs ont été abordés dans le questionnaire (tableau 1). Précisons que les pourcentages présentés se rapportent au nombre de personnes qui pratiquent l'activité en question et non à la population totale ${ }^{6}$. Les activités les plus pratiquées en Suisse sont tout d'abord la promenade $-72,6 \%$ des personnes qui déclarent faire des promenades, le font également en Suisse -, suivie de la fréquentation de patinoires $(71,4 \%)$, de piscines $(64,3 \%)$, de bars, restaurants et discothèques (64\%), de la pratique de sports et activités de plein-air $(62,6 \%)$ et de la participation à des activités culturelles (60\%). Les autres activités (cinéma, événements sportifs et sport en salle) sont pratiquées par la moitié des personnes concernées uniquement dans leur pays de résidence.

Pour l'analyse des pratiques d'achat, huit catégories de biens et services ont été retenues (tableau 1). À l'instar des loisirs, les chiffres présentés ne rendent pas compte de la fréquence des achats, mais indiquent uniquement le pays dans lequel ces pratiques spatiales prennent place. Ils se réfèrent par contre cette fois à l'ensemble de la population. La Suisse semble être attractive pour quelques produits en particulier. Il s'agit tout d'abord de l'essence qui représente le produit acheté par le plus grand nombre de personnes en Suisse $(66,6 \%)$. Les produits alimentaires attirent également beaucoup d'habitants de la zone frontalière $(62,5 \%)^{7}$ de même que les vêtements et articles de sports (58,4\%). À cette liste s'ajoutent les cigarettes. La proportion de sondés qui achètent des cigarettes en Suisse $(24,3 \%)$ semble de prime abord faible mais est en réalité très élevée si on la compare à la population estimée de fumeurs en France (environ $30 \%$ des 18-75 ans ${ }^{8}$ ). Les autres achats sont en retrait: entre un quart et un tiers des ménages achètent de temps à autre des meubles ou articles de décoration, des montres et bijoux, de l'électroménager/HIFI ou des cosmétiques en Suisse.

\section{Influence du lieu de travail}

Cette partie de l'analyse s'intéresse aux pratiques spatiales transfrontalières en distinguant trois groupes de population en fonction de leur statut d'activité et, le cas échéant, de leur lieu de travail. Le tableau 2 mentionne ainsi les pratiques 
transfrontalières des frontaliers, des actifs travaillant en France et des non-actifs. Ceci permet de mettre en lumière l'influence du lieu de travail sur les pratiques du fait des passages réguliers de la frontière, d'un pouvoir d'achat plus élevé ${ }^{9}$ et de certaines formes de socialisation grâce aux contacts avec des collègues suisses.

Concernant les réseaux sociaux, plus de la moitié des travailleurs frontaliers $(54,4 \%)$ ont des amis en Suisse contre 23,1\% des actifs employés en France et 31,5\% des non-actifs. Des différences encore plus prononcées sont observées en termes de vie associative. Ainsi, $59 \%$ des frontaliers qui fréquentent une ou plusieurs associations, sont membres d'au moins une association en Suisse. Cette proportion tombe à $25,4 \%$ pour les non-actifs et $22,6 \%$ pour les personnes actives en France.

D'importants écarts sont également constatés dans la pratique des loisirs entre les frontaliers et le reste de la population. Les plus grandes divergences concernent la participation à des événements sportifs en tant que spectateur (près de 30 points d'écarts avec les actifs français et plus de 50 points avec les non-actifs). De même, on trouve des différences marquées par rapport à la fréquentation des bars et autres établissements publics : 79,7 \% des frontaliers qui se rendent dans ce genre de lieu le font également en Suisse, contre $51,5 \%$ des actifs français et $47,9 \%$ des non-actifs. Ces deux activités pouvant se caractériser par des contacts sociaux plus importants, la socialisation des frontaliers (à travers leurs collègues et amis résidant en Suisse) exerce une influence sensible. Ils semblent ainsi adopter certaines pratiques (soutien à l'équipe sportive locale) ou fréquenter les mêmes bars que leurs collègues ou amis. La pratique de sport en salle et la fréquentation d'infrastructures ou d'événements culturels varient aussi fortement. Il est intéressant de noter que les loisirs pour lesquels les différences entre frontaliers et le reste de la population sont les plus petites et/ou non-significatives, sont des loisirs qui ne sont praticables qu'en Suisse dans cette région. Il n'y a en effet ni patinoire, ni piscine extérieure dans la zone frontalière française. Ceci signifierait que les non-frontaliers sont prêts à se rendre en Suisse pour les activités qu'ils ne peuvent pas pratiquer dans la zone frontalière française.

Pour ce qui est des habitudes de consommation en Suisse, des écarts sont observés pour tous les produits entre les frontaliers et le reste de la population (ils sont par ailleurs tous significatifs à l'exception de l'essence). Les plus grands concernent l'achat d'électroménager/HIFI (près de la moitié des frontaliers en achètent en Suisse contre $17,1 \%$ des actifs français et 14,9\% des non-actifs), de montres/bijoux et de vêtements/ articles de sports. Ces différences significatives peuvent s'expliquer par le pouvoir d'achat plus grand qui caractérise les travailleurs frontaliers.

Le lieu de travail influence donc globalement de manière considérable les pratiques spatiales. Les travailleurs frontaliers ont beaucoup plus tendance à pratiquer le territoire suisse que le reste de la population, et ce dans les trois catégories retenues (réseaux sociaux, loisirs, achats).

Tableau 2. Pratiques spatiales transfrontalières (frontaliers, actifs français, non-actifs).

\begin{tabular}{|l|l|l|l|l|}
\hline & Frontaliers & $\begin{array}{l}\text { Actifs } \\
\text { français }\end{array}$ & $\begin{array}{l}\text { Non- } \\
\text { actifs }\end{array}$ & Significativité* \\
\hline Réseaux sociaux : & & & & \\
\hline
\end{tabular}




\begin{tabular}{|c|c|c|c|c|}
\hline $\begin{array}{l}\text { Réseau d'amis en Suisse et en France ou } \\
\text { uniquement en Suisse }\end{array}$ & $54.4 \%$ & $23.1 \%$ & $31.5 \%$ & $* * *$ \\
\hline $\begin{array}{l}\text { Participation à des associations en Suisse } \\
\text { et en France ou uniquement en Suisse }\end{array}$ & $59.0 \%$ & $22.6 \%$ & $25.4 \%$ & $* * *$ \\
\hline \multicolumn{5}{|l|}{ Loisirs en Suisse } \\
\hline Promenade & $84.2 \%$ & $73.7 \%$ & $57.9 \%$ & $* * *$ \\
\hline Bar, restaurant, discothèque & $79.7 \%$ & $51.5 \%$ & $47.9 \%$ & $* * *$ \\
\hline $\begin{array}{l}\text { Théâtre, spectacle, concert, } \\
\text { musée, événement culturel }\end{array}$ & $74.1 \%$ & $54.5 \%$ & $44.1 \%$ & $* * *$ \\
\hline Cinéma & $60.8 \%$ & $34.9 \%$ & $43.7 \%$ & ** \\
\hline Piscine & $67.7 \%$ & $64.8 \%$ & $56.5 \%$ & N.S. \\
\hline Sport, activité de plein-air & $69.7 \%$ & $60.4 \%$ & $49.2 \%$ & * \\
\hline Evénement sportif (spectateur) & $68.1 \%$ & $38.8 \%$ & $17.8 \%$ & $* * *$ \\
\hline $\begin{array}{l}\text { Sport en salle } \\
\text { (par ex: squash, tennis, fitness) }\end{array}$ & $60.3 \%$ & $35.1 \%$ & $31.6 \%$ & * \\
\hline Patinoire & $78.8 \%$ & $71.4 \%$ & $50.0 \%$ & N.S. \\
\hline \multicolumn{5}{|l|}{ Achats en Suisse } \\
\hline Produits alimentaires & $76.4 \%$ & $49.4 \%$ & $57.4 \%$ & $* * *$ \\
\hline Essence & $71.7 \%$ & $65.9 \%$ & $61.6 \%$ & N.S. \\
\hline Cigarettes & $34.6 \%$ & $25.3 \%$ & $13.2 \%$ & $* * *$ \\
\hline Vêtements/articles de sport & $77.2 \%$ & $51.8 \%$ & $43.4 \%$ & *** \\
\hline $\begin{array}{l}\text { Produits liés à l'équipement de la maison } \\
\text { (meubles, décorations) }\end{array}$ & $49.6 \%$ & $31.8 \%$ & $20.7 \%$ & $* * *$ \\
\hline Montres/Bijoux & $50.0 \%$ & $21.2 \%$ & $15.3 \%$ & *** \\
\hline Électroménager/HIFI & $48.8 \%$ & $17.1 \%$ & $14.9 \%$ & $* * *$ \\
\hline Cosmétiques, bien-être, salon de coiffure & $34.6 \%$ & $18.2 \%$ & $15.7 \%$ & $* * *$ \\
\hline
\end{tabular}

Note : le test du chi-carré a été utilisé pour mesurer la significativité des différences entre les trois groupes de population. Les seuils de signification suivants ont été retenus : 0,05 (*), 0,01 (**) et 0,001 $(\star \star \star)$. N.S. signifie que la différence n'est statistiquement pas significative. 


\section{Logiques d'action}

29 Cette partie cherche à mettre en lumière les mécanismes expliquant les pratiques spatiales transfrontalières. Nous utilisons à cette fin des citations tirées du questionnaire. La présentation des résultats est structurée selon les trois logiques mentionnées dans la partie théorique : la logique économique et calculatrice, la logique utilitaire et la logique socio-culturelle. Les facteurs que l'on peut rattacher à ces logiques constituent tantôt des freins tantôt des incitations au franchissement de la frontière.

30 La logique économique renvoie aux critères de coûts et de taux de change. La frontière induit dans ce cas un différentiel de prix. De manière générale, le coût de la vie n'est pas un élément attractif pour la Suisse et les prix des biens et services y sont considérés comme élevés :

"Le prix de certains loisirs est exorbitant. Le cinéma par exemple." (Homme, 30 ans, travailleur frontalier)

31 Quelques produits font toutefois exception comme l'essence et les cigarettes. Il s'agit de produits identiques de part et d'autre de la frontière et qui ne diffèrent qu'en termes de prix en raison d'un niveau de taxation différent. Il s'agit donc là d'une consommation strictement liée à l'effet frontière.

L'écart entre les prix dépend également du taux de change en vigueur. Ces dernières années, le renforcement du franc suisse vis-à-vis de l'euro a rendu le coût de la vie en Suisse encore plus cher. C'est particulièrement le cas pour les personnes ne touchant pas leur salaire en francs suisses ${ }^{10}$ :

"J'étais une fidèle consommatrice en Suisse il y a quelques années, mais avec le taux de change, rien n'est plus attractif." (Femme, 55 ans, active en France)

À partir d'un certain niveau, la dépréciation de l'euro face au franc suisse pourrait avoir également une influence sur les achats de produits en Suisse motivés par la seule différence de prix (essence et cigarettes). Il semblerait toutefois que le taux de change revêt une importance moindre pour ce qui est des loisirs :

"Mes loisirs n'ont pas de frontières et encore moins un taux de change. Quand je patine sur le Doubs, je me moque de savoir de quel côté je suis." (40 ans, femme au foyer)

"Le taux de change n'est pas forcément important au niveau des loisirs." (Homme, 25 ans, travailleur frontalier)

Ainsi, bien que le prix et le taux de change aient une grande importance dans les pratiques spatiales transfrontalières, ils ne sont de loin pas les seuls facteurs à entrer en jeu. La deuxième logique, utilitaire et pratique, est également à prendre en compte. Elle met en évidence des différentiels d'offre de part et d'autre de la frontière.

Certains résidents français de la zone frontalière se rendent ainsi en Suisse pour trouver des produits spécifiques à la Suisse ou qu'ils estiment de meilleure qualité :

"Je fais mes achats dans les deux pays : en Suisse l'offre est différente et me permet de varier les produits." (Femme, 45 ans, travailleuse frontalière)

"J'achète certains produits de meilleure qualité en Suisse comme le chocolat, le poisson frais ou l'électroménager, sinon le reste je l'achète en France." (Femme, 50 ans, travailleuse frontalière)

Un aspect supplémentaire d'ordre utilitaire est lié au contexte géographique. Malgré des prix plus élevés, les magasins suisses sont fréquentés par des résidents français pour des achats ciblés (et non pas pour des achats courants) de produits que l'on ne trouve pas de 
l'autre côté de la frontière. Il s'agit donc ici essentiellement d'un effet d'agglomération et de centralité. En l'absence de pôles urbains français d'importance, les habitants de Morteau et Villers-le-Lac se tournent vers les villes helvétiques (en premier lieu La Chaux-de-Fonds) pour bénéficier d'une offre plus développée en services et loisirs :

"Les grandes villes françaises sont éloignées de Villers-le-Lac, c'est la principale raison qui fait de nous des consommateurs des magasins suisses." (Femme, 25 ans, travailleuse frontalière)

"Pour moi il n'y a pas de frontière. Je choisis le lieu de mes loisirs selon le côté pratique. Je vais en Suisse quand l'offre est meilleure ou si c'est plus près." (Travailleur frontalier)

"Les sorties en Suisse sont surtout dirigées par l'impossibilité de pratiquer en France ou par la proximité du territoire suisse." (Homme, 35 ans, actif en France)

"Je ne pratique pas mes loisirs selon le pays, mais plutôt selon ce qui m'intéresse. Je trouve qu'il y a plus de choses à faire du côté suisse qu'à Villers-le-Lac ou Morteau." (Homme, 35 ans, actif en France)

Parfois, la localisation des pratiques spatiales d'un côté ou de l'autre de la frontière relève de considérations essentiellement pragmatiques :

"Nous fréquentons des associations et clubs en France. C'est plus pratique avec les horaires d'école de nos enfants en France." (Femme, 45 ans, travailleuse frontalière) "Je privilégie le côté pratique : c'est plus simple de faire du fitness en Suisse la semaine après le travail, mais par contre pour le football, je le pratique le week-end en France." (Homme, 25 ans, travailleur frontalier)

La troisième logique regroupe des facteurs socio-culturels tels que l'habitude, les préférences et la question du degré d'information quant au pays voisin. Il s'agit ici d'un différentiel de connaissances. Pour les loisirs comme pour les achats, une majorité des sondés déclarent exercer ces activités dans leur pays de domicile par habitude. De même, la préférence (de pratiquer ses loisirs en France ou de consommer des produits français) est également invoquée. On peut discerner à travers l'habitude et les préférences, un effet frontière dans le sens d'une barrière de nature psychologique.

Malgré la défonctionnalisation de la frontière qui permet un franchissement aisé, il semble que les informations circulent encore de manière relativement difficile. En effet, le manque de connaissance des offres de loisirs, culturelles et commerciales, ou plus généralement du territoire suisse, n'incite pas les habitants à traverser la frontière :

"Nous aimons beaucoup sortir en Suisse, mais nous ne sommes pas toujours bien informés de ce qui s'y passe." (Homme, 50 ans, actif en France)

"Le Locle et La Chaux-de-Fonds sont beaucoup plus proches que Besançon ou Montbéliard. Mais je manque d'informations et d'habitude pour m'y rendre régulièrement." (Femme, 50 ans, active en France)

Cet effet peut être lié à l'absence d'un réseau social de l'autre côté de la frontière ou à la difficulté d'en créer un :

"Nous ressentons vivement une mentalité anti-frontalier surtout depuis la crise. Dommage car nous nous plaisons bien en Suisse" (Homme, 50 ans, travailleur frontalier)

"Il est très difficile de se faire des amis en Suisse, on est des collègues et non des amis." (Homme, 55 ans, à la recherche d'un emploi)

"Je pense qu'un frontalier reste un frontalier vis-à-vis d'un Suisse." (Homme, 40 ans, travailleur frontalier)

41 Les facteurs socio-culturels n'ont pas uniquement comme effet de freiner les pratiques spatiales transfrontalières. En effet, comme nous l'avons constaté, travailler en Suisse 
augmente nettement la probabilité de s'y rendre pour d'autres activités et pour certains, la frontière semble s'être estompée :

"Je pratique mes loisirs avec des amis et des collègues suisses qui m'ont fait des découvrir certains lieux que j'apprécie beaucoup". (Femme, 54 ans, travailleuse frontalière)

"Les amis qu'ils soient suisses ou français, je ne fais pas de différences. Si nous sommes amis, ce que nous avons des points communs, la nationalité n'a pas d'importance." (Femme, 60 ans, travailleuse frontalière)

\section{Conclusion}

42 Cet article aborde la question des pratiques spatiales transfrontalières lors du temps libre (soit hors travail ou formation) et liées à la vie quotidienne ou de nature routinière (soit hors voyages ou activités ponctuelles). Ces pratiques constituent des indicateurs du degré d'intégration fonctionnelle d'une région, l'Arc jurassien franco-suisse, qui se caractérise par une intégration formelle élevée. Sur la base d'une enquête par questionnaire auprès d'habitants de deux communes françaises proches de la frontière, l'objectif consistait, d'une part, à quantifier l'importance des pratiques spatiales transfrontalières et, d'autre part, à identifier les logiques et facteurs jouant le rôle de frein ou d'incitation au franchissement de la frontière. Plusieurs enseignements d'ordre général peuvent être tirés sur la base de cette étude de cas par rapport au développement transfrontalier.

43 Il est difficile de tirer des conclusions précises sur l'intensité des pratiques spatiales transfrontalières en l'absence de base de comparaison dans le temps (avec par exemple la situation de Morteau et Villers-le-Lac il y a quelques années, notamment avant l'entrée en vigueur des accords sur la libre circulation des personnes entre la Suisse et l'Union Européenne ${ }^{11}$ ) et dans l'espace (avec par exemple la situation dans d'autres contextes territoriaux similaires traversés ou non par une frontière nationale). Néanmoins, notre étude a montré que les franchissements de la frontière ne sont pas uniquement liés au marché du travail mais se retrouvent dans les réseaux sociaux, les loisirs et les achats.

Les pratiques spatiales transfrontalières les plus courantes - mesurées par la proportion de la population interrogée qui les réalise en partie en Suisse - sont liées à certaines activités sportives - telles que la promenade et la fréquentation d'équipements spécifiques (patinoire, piscine), etc. -, à la vie nocturne et aux activités culturelles. Les achats se portent en premier lieu sur l'essence, certains produits alimentaires, les vêtements et les cigarettes. Les pratiques spatiales transfrontalières sont en revanche moins intenses pour l'achat des autres biens et services. Le réseau social - cercle d'amis et vie associative - ne franchit pas la frontière pour $40 \%$ des personnes interrogées.

Ces pratiques répondent à trois logiques. La première est une logique économique qui se base sur le différentiel de prix induit par la frontière (et lié au coût de la vie, au système de taxation, au taux de change). Les ménages adaptent une partie de leurs pratiques notamment en termes d'achat - en fonction de calculs économiques comparatifs.

La seconde logique est d'ordre utilitaire et met en exergue un différentiel d'offre. L'absence de pôles urbains français, la fonction résidentielle des communes françaises proches de la frontière et la taille des centres urbains suisses constituent autant de facteurs qui induisent un effet d'agglomération ou de centralité. L'offre, plus large et diversifiée du côté suisse, couplée avec une distance raisonnable, attire de nombreux habitants de la zone frontalière française pour des achats ou loisirs. Il ne s'agit pas ici 
d'un effet frontière au sens strict mais davantage d'une conséquence du contexte territorial. Au contraire, sans la frontière, on peut estimer que les interactions entre les différentes communes étudiées seraient encore plus développées.

La troisième logique regroupe des éléments de nature socio-culturelle tels que les préférences ou l'habitude. Elle est liée à un différentiel de connaissances ou d'informations et représente un avatar supplémentaire de l'effet frontière. La différence d'intensité des pratiques spatiales transfrontalières entre les habitants de France employés en Suisse et le reste de la population est à cet égard manifeste (comme l'avaient également relevé Bolzman et Vial, 2007). En raison de fréquents trajets de l'autre côté de la frontière, des contacts entretenus avec des collègues suisses voire de la constitution d'un groupe d'amis mais aussi du fait de leur pouvoir d'achat élevé, les travailleurs frontaliers exercent davantage d'activités en Suisse que les travailleurs employés en France ou que les non actifs. Si cela peut paraître évident par rapport à la littérature sur la socialisation, ce résultat bat en brèche le stéréotype du travailleur frontalier uniquement motivé par un comportement opportuniste basé sur le différentiel de salaire. De nombreux sondés pratiquent en effet leurs loisirs en Suisse, y consomment ou y ont développé des réseaux sociaux.

Dans l'ensemble, cette étude montre que l'effet frontière peut freiner ou inciter certaines pratiques transfrontalières et que son impact dépend du contexte territorial. Dans notre étude de cas, s'il apparaît que la frontière "met de la distance dans la proximité" (Groupe frontière, 2004) et génère un espace spécifique à "l'horizon tronqué" (Wackermann, 2005), elle n'empêche pas de nombreuses pratiques transfrontalières. À un niveau élevé d'intégration formelle répond un degré d'intégration fonctionnelle non négligeable et qui semble en croissance par rapport aux dernières décennies. Derrière cette intégration fonctionnelle se cachent néanmoins des pratiques et des vécus variables. En d'autres termes, la frontière n'est pas seulement une construction politique mais également une construction sociale divergente selon les individus et groupes de population.

\section{BIBLIOGRAPHIE}

ANDERSON J. and O'DOWD L. (1999), "Borders, border regions and territoriality: contradictory meanings and changing significance", Regional studies, 33, 7, pp. 593-604.

BLAKE G. (2000), "State limits in the early twenty-first century: Observations on form and function", Geopolitics, 5, 1, pp. 1-18.

BOLZMAN C. et VIAL M. (2007), Migrants au quotidien : les frontaliers. Pratiques, représentations et identités collectives, Zurich, Seismo.

CHEVAILLER J.C. (1990), Les travailleurs frontaliers francs-comtois en Suisse, Besançon, CETAP/ Université de Franche-Comté.

CREDIT SUISSE (2011), Retail Outlook 2012. Faits et tendances, Zurich, Credit Suisse Economic Research. 
CREVOISIER O., MOINE A., CORPATAUX J., QUIQUEREZ F. et SIGNORET P. (2006), Impact de l'accord bilatéral relatif à la libre circulation des personnes sur la région transfrontalière du canton de Neuchâtel et du département $d u$ Doubs, Neuchâtel, Groupe de recherche en économie territoriale.

DAVEAU S. (1965), "Déclin des rapports frontaliers avec la France aux limites du canton de Neuchâtel”, Bulletin de la Société neuchâteloise de géographie, 52, pp. 305-313.

MACHADO DE OLIVEIRA T.C. (2009), “Frontières en Amérique latine : réflexions méthodologiques", Espaces et sociétés, 3, 138, pp. 19-33.

DIENER A. C. and HAGEN J. (2009), “Theorizing Borders in a 'Borderless World': Globalization, Territory and Identity”, Geography Compass, 3, 3, pp. 1196-1216.

GARUFO F. (2009), “Travail frontalier et segmentation du marché de l'emploi horloger : le cas de Tissot SA (1960-1980)”, Géo-Regards, 2, pp. 23-37.

GIDDENS A. (1984), The Constitution of Society. Outline of the Theory of Structuration, Cambridge, Polity Press.

GROUPE FRONTIÈRE (2004), “La frontière, un objet spatial en mutation”, Espacestemps.net, http:// www.espacestemps.net/document842.html.

HELD D. and MCGREW A. (eds.) (2003), The global transformations reader: an introduction to the globalization debate, Cambridge, Polity Press.

JUAN S. (1997), Les sentiers du quotidien : rigidité, fluidité des espaces sociaux et trajets routiniers en ville , Paris, L'Harmattan.

MOINE A. (2003), “Représentation et compréhension de la systémogénèse d'un espace de production transfrontalier”, Mappemonde, www.mgm.fr/PUB/Mappemonde/M303/Moine.pdf.

MUNZ H. (2011), “Les (im)mobiles frontières du patrimoine horloger dans l'Arc jurassien”, GéoRegards, 4, à paraître.

NELLES J. and WALTHER O. (2011), “Changing European borders: from separation to interface? An introduction”, Articulo - Journal of Urban Research, 6.

NEWMAN D. (2006), “The lines that continue to separate us: borders in our 'borderless' world”, Progress in Human Geography, 30, 2, pp. 143-161.

O'BRIEN R. (1992), Global financial integration: the end of geography, Londres, Royal Institute of International Affairs.

OHMAE K. (1990), The borderless world. Power and strategy in the interlinked economy, London, Collins.

OSTAJ (Observatoire Statistique Transfrontalier de l'Arc Jurassien) (2009), Une comparaison des salaires entre la Franche-Comté et l'Arc jurassien suisse, Besançon et Neuchâtel, OSTAJ.

REITEL B. (2007), "Les agglomérations transfrontalières : des systèmes urbains en voie d'intégration ? : les espaces urbains de la 'frontière' du territoire français", Geographica Helvetica, 62, pp. 4-15.

RÉRAT P., MOINE A., GERTSCH K. et SIGNORET P. (2011), “La mobilité résidentielle transfrontalière dans l'Arc jurassien franco-suisse”, Géo-Regards, 4, pp. 153-168.

RÉRAT P. et WASTL-WALTER D. (éd.) (2011), “Développement transfrontalier et mobilités (numéro spécial)", Géo-Regards, 4.

SOHN C. et WALTHER O. (2009), "Métropolisation et intégration transfrontalière : le paradoxe luxembourgeois", Espaces et sociétés, 3, 138, pp. 51-67. 
WACKERMANN G. (2005), Les frontières dans un monde en mouvement, Paris, Editions Ellipses.

WASTL-WALTER D. (2009), “Borderlands”, in KITCHIN R., THRIFT N. (eds.), International

Encyclopedia of Human Geography, 1, Oxford, Elsevier, pp. 332-339.

WASTL-WALTER D. (2011), The Ashgate research companion to border studies, Farnham, Ashgate.

\section{NOTES}

1. L'accord de Schengen par exemple supprime les contrôles d'identité systématiques aux frontières, favorisant ainsi la mobilité en Europe (y compris avec la Suisse). L'accord de libre circulation des personnes règlemente quant à lui le franchissement de la frontière, mais aussi le séjour et la pratique d'une activité économique dans un pays signataire. Cet accord prévoit notamment un abandon de la préférence de la main-d'œuvre indigène lors de l'embauche et élargit les conditions d'obtention d'un permis de travail frontalier. Bien que ces deux accords témoignent d'une importante intégration formelle, il convient d'apporter une nuance concernant les dispositions réglementant l'importation de biens pour la consommation personnelle pour les habitants des zones frontalières car celles-ci sont plutôt restrictives. Toutefois, au vu des observations faites dans la partie empirique, elles n'empêchent aucunement la consommation transfrontalière (la fréquence des contrôles douaniers n'étant pas perçue comme dissuasive).

2. Depuis Morteau, il faut environ 1 heure pour se rendre à Besançon, 35 minutes pour Pontarlier, 30 minutes pour La Chaux-de-Fonds et 20 minutes pour Le Locle.

3. Un total de 670 questionnaires a été complété alors que 147 questionnaires sont revenus sans avoir pu être délivrés à leur destinataire (décès, déménagement, etc.).

4. Les sondés disposaient du choix de réponses suivant : "en France uniquement", "la plupart en France", "en France et en Suisse", "la plupart en Suisse", "uniquement en Suisse". Les deux premières réponses ont été agrégées sous le label "uniquement en France", les trois dernières sous la dénomination "en France et en Suisse ou uniquement en Suisse".

5. Cinq types d'associations ont été pris en compte: club de sport, syndicat/association lié au travail, groupe musical/chorale/théâtre, association à but social/culturel/environnemental, groupement à but religieux/spirituel.

6. Les chiffres entre parenthèses donnent quant à eux une indication du nombre total de personnes concernées par une activité.

7. Précisons toutefois que pour près d'un tiers d'entre eux les achats en Suisse sont ponctuels (moins d'une fois par mois) et concernent certains produits spécifiques (comme le chocolat).

8. International Tobacco Control, 2009, Projet d'évaluation des politiques publiques de lutte antitabac. Rapport national ITC France.

9. En 2006, un frontalier travaillant dans l'Arc jurassien suisse perçoit un salaire horaire brut supérieur de 50 \% à celui d'un salarié travaillant en Franche-Comté (OSTAJ 2009).

10. À l'inverse, il devient plus intéressant pour les résidents suisses de se rendre dans les pays limitrophes, ce qui a provoqué une très forte croissance du "tourisme d'achat" (Credit Suisse, 2011).

11. À cet égard, il est intéressant de lire certains travaux historiques qui montrent l'importance des pratiques spatiales transfrontalières avant le $\mathrm{XX}^{\mathrm{e}}$ siècle, siècle qui a vu l'instauration d'un contrôle de plus en plus poussé des frontières du fait notamment des deux guerres mondiales et de la crise des années trente. Pour le cas de l'Arc jurassien, voir Daveau (1965). 


\section{RÉSUMÉS}

La construction européenne a redéfini la signification et les fonctions des frontières nationales. Cet article s'intéresse à cette mutation sous l'angle des pratiques des habitants de l'Arc jurassien franco-suisse. Une enquête par questionnaire a permis de mesurer l'intensité de certaines pratiques spatiales transfrontalières réalisées pendant le temps libre (achats, loisirs, etc.) et d'en déterminer les logiques sous-jacentes. L'effet frontière se manifeste sous la forme d'un différentiel de prix (coût de la vie, taux de change, taxation de certains produits) et d'un différentiel de connaissances (manque d'informations sur le pays voisin, habitude, etc.) qui freinent ou incitent le franchissement de la frontière. L'effet frontière est toutefois atténué dans le contexte territorial étudié par un effet de centralité impliquant un différentiel d'offre (attraction des communes françaises à vocation résidentielle par les centres urbains helvétiques). La combinaison de ces effets explique l'intensité et l'orientation des pratiques spatiales transfrontalières.

The European integration has been redefining the meaning and functions of national borders. This paper addresses this mutation from the perspective of the inhabitants' spatial practices in the French-Swiss Jura region. Through a questionnaire survey we have measured the intensity of cross-border spatial practices done in the free time (purchasing, leisure, etc.) and determined the underlying logics. The border effect appears in the form of a price differential (cost of living, exchange rate, taxation of some goods) and a knowledge differential (lack of information on the neighbouring country, habits, etc.) that curb or stimulate border crossing. The border effect is however mitigated in the spatial context under study by a centrality effect involving a supply differential (attraction of residential French municipalities by Swiss urban centres). The combining of these effects explains the intensity and the direction of cross-border spatial practices.

\section{INDEX}

Keywords : border, spatial practices, borderland development, logic of action, France, Switzerland

Mots-clés : frontière, pratiques spatiales, développement transfrontalier, logique d'action, France, Suisse

\section{AUTEURS}

\section{YANN DUBOIS}

Institut de géographie, Université de Neuchâtel, Suisse, yann.dubois@unine.ch 


\section{PATRICK RÉRAT}

Institut de géographie et Institut de sociologie, Université de Neuchâtel, Suisse, patrick.rerat@unine.ch 